\title{
Alteraçōes Endometriais em Pacientes com Câncer de Mama Tratadas com Tamoxifeno
}

\author{
Endometrial Findings in Patients with Breast Cancer Using Tamoxifen \\ Francisco Edson de Lucena Feitosa, Sérgio Ferreira Juaçaba, \\ Francisco das Chagas Medeiros
}

\begin{abstract}
RESUMO
Objetivos: avaliar sensibilidade, especificidade, valor preditivo positivo e negativo e razão de probabilidade da ultra-sonografia transvaginal e da histeroscopia quando comparadas com o exame histopatológico do endométrio em mulheres com câncer de mama tratadas com tamoxifeno.

Método: estudo transversal, com trinta mulheres, em que se avaliou a ecogenicidade do eco endometrial e a sua espessura por meio de ultra-sonografia transvaginal. Realizou-se histeroscopia que foi descrita como normal (endométrio normal ou atrófico) ou anormal (espessamento, pólipo, mioma, sinéquia). Mediante biópsia endometrial foi obtido material para exame histopatológico e os resultados foram considerados normais (endométrio de maturação irregular e/ou atrofia) ou anormais (pólipos, hiperplasia simples ou complexa, miomas ou carcinoma endometrial).

Resultados: a prevalência geral de alterações endometriais foi de 36,6\%. Os achados mais freqüentes foram a atrofia cística $(46,6 \%)$ e os pólipos (26,6\%). Por meio de curva ROC determinou-se que o melhor ponto de corte da medida da espessura do endométrio foi $8 \mathrm{~mm}$. Esta medida apresentou sensibilidade de 72,7\%, especificidade de 78,9\%, valor preditivo positivo de $66,6 \%$, valor preditivo negativo de $83,3 \%$ e razão de probabilidade de 3,4 . A histeroscopia apresentou sensibilidade de 90,9\%, especificidade de 68,4\%, valor preditivo positivo de $62,5 \%$, valor preditivo negativo de $92,8 \%$ e razão de probabilidade de 2.8 .

Conclusão: os achados mais freqüentes foram a atrofia cística e os pólipos. A ultra-sonografia transvaginal apresentou maior taxa de falso-positivo $(42,1 \%)$, utilizando ponto de corte da espessura do endométrio de $5 \mathrm{~mm}$, porém melhorou-se a acurácia quando se utilizou a medida de $8 \mathrm{~mm}$ obtido pela confecção de curva ROC.
\end{abstract}

PALAVRAS-CHAVE: Tamoxifeno. Endométrio. Ultra-sonografia transvaginal. Histeroscopia. Mama: câncer.

\section{Introdução}

O tamoxifeno foi inicialmente empregado com finalidade contraceptiva e, posteriormente, como indutor da ovulação. O mecanismo primário de ação do fármaco, como também o de outros antiestrogênios, seria o antagonismo à molécula

Maternidade-Escola Assis Chateaubriand da Universidade Federal do Ceará

Hospital do Câncer do Ceará

Correspondência:

Francisco Edson de Lucena Feitosa

Av. Santos Dumont, 6915 apto 601 - Papicu

60190-800 - Fortaleza - Ce

e-mail: edsonlucena@secrel.com.br do estrogênio nos seus receptores específicos ${ }^{1,2}$. Porém, diversos autores referiram que o mecanismo de ação do tamoxifeno é complexo ${ }^{3,4}$, e ainda não está adequadamente elucidado ${ }^{4}$.

Sabe-se que os antiestrogênios comportamse como antagonistas puros ou agonistas/antagonistas parciais, dependendo da espécie animal ou da célula-alvo, o que dificulta a elucidação do seu mecanismo de ação ${ }^{5}$.

O primeiro relato da utilização do tamoxifeno no tratamento do câncer de mama foi o de Cole et al. ${ }^{6}$. Em 1973, o "Committee on the Safety of Medicines", no Reino Unido, aprovou o tamoxifeno como tratamento adjuvante do câncer de mama 
e, somente em 30 de dezembro de 1977, foi aprovado pela "FDA - Food and Drug Administration", para o mesmo fim ${ }^{7}$. Em seguida, estudos clínicos e laboratoriais com antiestrogênios foram realizados e atualmente é medicação hormonal de primeira linha para tratar pacientes com essa neoplasia.

O tamoxifeno foi, originalmente, selecionado para o uso terapêutico devido à sua eficácia e baixa incidência de efeitos colaterais, em relação a outros antiestrogênios não esteróides. Entretanto, alguns autores referiram sua influência na gênese de lesões endometriais, possibilitando, desse modo, maior risco de neoplasia de endométrio ${ }^{7-10}$.

Tendo em vista esses achados, a monitorização do endométrio das pacientes tratadas com tamoxifeno, com o intuito de diagnosticar precocemente essas lesões, foi recomendada, seja por histeroscopia ${ }^{11,12}$, por ultra-sonografia ${ }^{13,14}$, por curetagem uterina ou por biópsia endometrial ${ }^{14}$. Outros, entretanto, criticaram a sensibilidade dos métodos ultra-sonográficos, mesmo se acompanhados da dopplervelocimetria ${ }^{15}$.

Porém não existe consenso na literatura sobre a ação do tamoxifeno no endométrio de mulheres na pós-menopausa com câncer de mama, bem como sobre a melhor forma de rastreamento das alterações endometriais.

Assim, foi objeto de interesse neste estudo avaliar e acompanhar, prospectivamente, um grupo de pacientes que completaram cinco anos de uso de tamoxifeno com o intuito de aquilatar a sua ação, bem como avaliar eventuais anormalidades que poderiam ocorrer na mucosa uterina, por meio da ultra-sonografia e da histeroscopia.

\section{Pacientes e Métodos}

Estudaram-se de forma prospectiva $30 \mathrm{mu}-$ lheres com câncer de mama, menopausadas, assintomáticas, selecionadas aleatoriamente, usuárias de tamoxifeno há cinco anos, assistidas no Hospital do Câncer do Ceará no período de janeiro de 2000 a julho de 2001.

Este estudo foi previamente aprovado pela Comissão de Ética Médica da Universidade Federal do Ceará.

Em todas as pacientes, após apresentação e assinatura do termo de consentimento pós-informação, foram realizados anamnese, ultra-sonografia pélvica e transvaginal, histeroscopia, biópsia endometrial e estudo anatomopatológico do material obtido da cavidade uterina.

Adotaram-se os seguintes critérios de exclu- são: uso de tamoxifeno por menos de cinco anos, sintomatologia ginecológica, principalmente sangramento transvaginal, menacme ou pré-menopausa, ausência de útero, não-realização de qualquer dos métodos propedêuticos, impossibilidade do estudo histopatológico e fatores que impedissem a feitura da ultra-sonografia transvaginal ou a coleta de material da cavidade uterina para estudo anatomopatológico.

Realizou-se ultra-sonografia pélvica e transvaginal com equipamento da marca Siemens, modelo Sonoline Versa-Pro, dotado de transdutores abdominal convexo de $3,5 \mathrm{MHz}$ e transvaginal de $6,5 \mathrm{MHz}$ de freqüência, multifreqüenciais, e Doppler colorido triplex.

Avaliou-se a ecotextura uterina, assim como a ecogenicidade do eco endometrial e a medida de sua espessura em corte longitudinal, no maior diâmetro ântero-posterior, desde a interface ecogênica da junção endométrio-miométrio, de um lado ao outro.

Para fazer a histeroscopia, empregou-se o histeroscópio de Hamou II, marca Storz, com ótica de $250 \times 4 \mathrm{~mm}$ e camisa diagnóstica de 245 x $5 \mathrm{~mm}$. Usou-se sistema de iluminação com fonte de Xenon 175. A expansão da cavidade uterina foi obtida por meio de solução fisiológica a $0,9 \%$. A visão intracavitária inicialmente foi panorâmica, dirigindo-se, a seguir, à região fúndica, cornual esquerda, cornual direita e parede anterior, lateral esquerda, posterior e lateral direita até o orifício interno do colo. O achado histeroscópico foi descrito como normal (endométrio normal ou atrófico) ou anormal (espessamento, pólipo, mioma, sinéquia).

As duas formas propedêuticas foram realizadas na Maternidade-Escola Assis Chateaubriand da Universidade Federal do Ceará, por profissionais que não tinham conhecimento prévio dos resultados obtidos pelo exame inicial.

Durante o exame histeroscópico, as áreas consideradas suspeitas ou de interesse foram biopsiadas. O material obtido foi depositado em frascos que continham solução de formol a 10\% e depois incluído em parafina. Os blocos foram cortados com micrótomo, sendo as lâminas coradas pela hematoxilina e eosina.

A análise destes tecidos foi efetuada pelos patologistas do Departamento de Patologia e Medicina Legal da Universidade Federal do Ceará. Os resultados foram considerados normais, se fosse encontrado endométrio de maturação irregular e/ ou atrofia, ou anormais se fossem encontrados pólipos, hiperplasia simples com ou sem atipias, hiperplasia complexa com ou sem atipias, miomas ou carcinoma endometrial.

Os resultados foram analisados com o auxí- 
lio do software Epi-Info 6.0. Utilizou-se o teste exato de Fisher para comparação entre duas proporções. Determinaram-se a sensibilidade, especificidade, valores preditivos positivo e negativo e razão de probabilidade, para avaliar a acurácia dos métodos. Foi confeccionada a curva ROC (receiver operator characteristic curve), plotando-se a taxa de verdadeiro-positivos (sensibilidade) contra a taxa de falso-positivos (1 - especificidade) ao longo de uma faixa de pontos de corte, para expressar a relação entre a sensibilidade e a especificidade.

\section{Resultados}

Com relação à idade das pacientes, a média encontrada foi de 53 anos, com minima de 39 anos, máxima de 74 anos e moda de 50 anos.

A média do volume uterino foi de $74,5 \mathrm{~cm}^{3}$, com volume mínimo e máximo, respectivamente, de 15 e $176 \mathrm{~cm}^{3}$. A espessura endometrial apresentou moda de $4 \mathrm{~mm}$, mínima de $2,2 \mathrm{~mm}$ e máxima de $22 \mathrm{~mm}$.

Na Figura 1 se encontra a curva ROC onde se observa que o melhor ponto de corte da espessura do endométrio, para o presente estudo, é $8 \mathrm{~mm}$, pois é o local que mais se aproxima do canto superior esquerdo do gráfico.

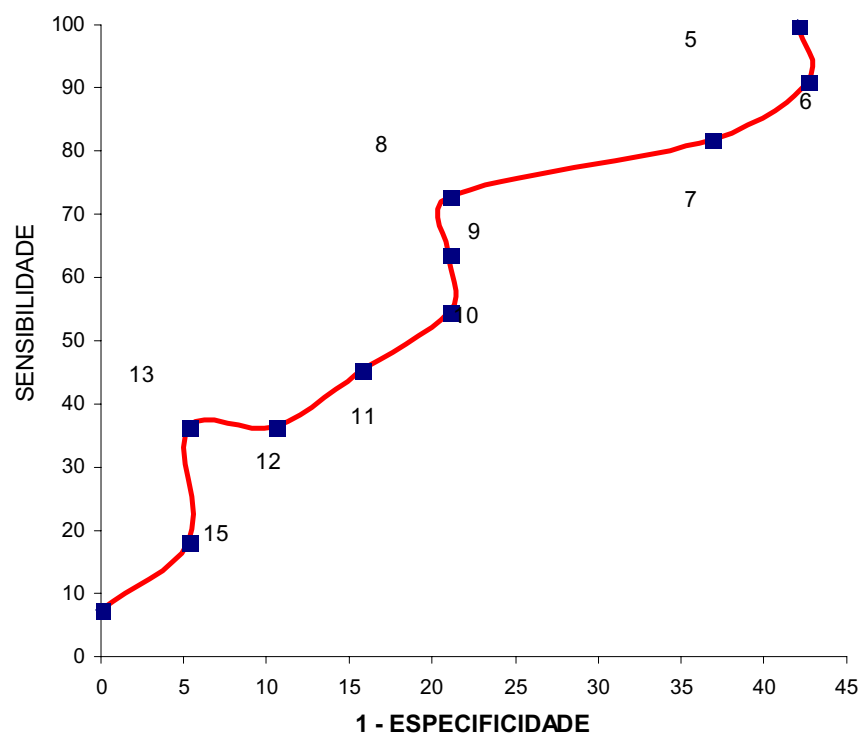

Figura 1 - Curva ROC, que mostra o contrabalanço entre sensibilidade e especificidade, demonstrando como melhor ponto de corte encontrado a medida de $8 \mathrm{~mm}$, pois é a que mais se aproxima do canto superior esquerdo do gráfico.

A sensibilidade, a especificidade, o valor preditivo positivo, o valor preditivo negativo, a acurácia e a razão de probabilidade da ultra-sonografia transvaginal para medida da espessura do endométrio estão anotados na Tabela 1, comparados com o resultado do exame histopatológico.

Tabela 1 - Sensibilidade, especificidade, valor preditivo positivo e negativo e razão de probabilidade da ultra-sonografia transvaginal em detectar alterações endometriais quando se adotou a medida de $8 \mathrm{~mm}$ do endométrio.

\begin{tabular}{lccc}
\hline Ultra-sonografia & \multicolumn{2}{c}{ Exame Histopatológico } & \\
\hline Anormal & Anormal & Normal & Total \\
Normal & 8 & 4 & 12 \\
Total & 3 & 15 & 18 \\
\hline
\end{tabular}

Sensibilidade $=72,7 \%$

Especifidade $=78,9 \%$

Valor preditivo positivo $=66,6 \%$

Valor preditivo negativo $=83,3$

Acurácia $=76,6 \%$

Razão de probabilidade $=3,45$

Os resultados da sensibilidade e especificidade obtidos avaliando-se a medida da espessura do endométrio ao longo de uma faixa de ponto de corte encontram-se na Figura 2. A medida de $8 \mathrm{~mm}$ apresenta o melhor contrabalanço entre sensibilidade e especificidade (seta vermelha).

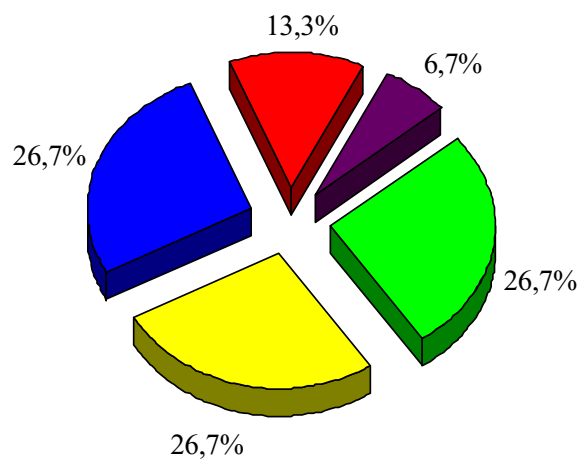

$\square$ Normal

Espessamento

Pólipo

Atrófico

Espessamento + pólipo

Figura 2 - Distribuição dos achados da histeroscopia.

Identificam-se na Figura 2 os resultados da histeroscopia, tendo sido considerada normal em $40 \%$ e anormal em $60 \%$ dos casos. Nestes, $26,6 \%$ representavam espessamento endometrial, 20\% pólipos e $6,6 \%$ a associação pólipo com espessamento endometrial.

Comparando-se, por meio do teste exato de Fischer, os resultados da histeroscopia com os resultados da ultra-sonografia, quando se adota como limite de normalidade a medida da espessura do endométrio de $5 \mathrm{~mm}$, encontra-se diferença significante $(p<0,05)$. Porém, quando se compara a histeroscopia com a espessura do endométrio de $8 \mathrm{~mm}$ o mesmo não ocorreu $(\mathrm{p}>0,05)$.

Está representada na Figura 3 a comparação da sensibilidade, especificidade, valor preditivo 
positivo, valor preditivo negativo e razão de probabilidade da ultra-sonografia transvaginal utilizando espessura do endométrio de $8 \mathrm{~mm}$ e a histeroscopia.

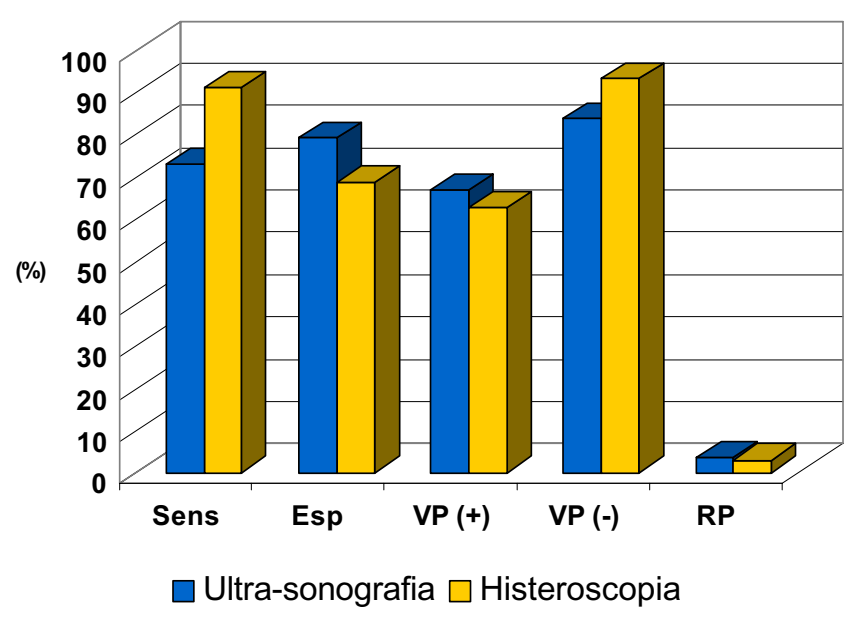

Figura 3 - Comparação da sensibilidade (Sens), especificidade (Esp), valor preditivo positivo (VP+), valor preditivo negativo (VP-) e razão de probabilidade (RP) da ultrasonografia, utilizando ponto de corte da medida da espessura do endométrio de $8 \mathrm{~mm}$, e da histeroscopia.

A análise histopatológica revelou endométrio normal em $63,3 \%$ dos casos e endométrio anormal em $36,7 \%$ dos casos. Destes, $23,4 \%$ eram representados por pólipos, 3,3\% por pólipo com endométrio atrófico, $3,3 \%$ por mioma submucoso e 6,7 por hiperplasia cística simples (Figura 4).

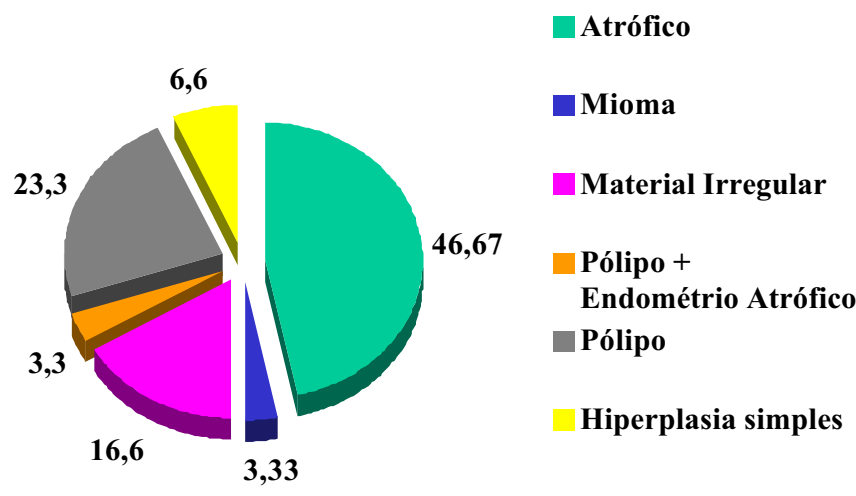

Figura 4 - Distribuição dos achados do exame histopatológico.

\section{Discussão}

O câncer de mama tem enorme impacto sobre a saúde feminina. A sua incidência varia consideravelmente entre os países. É grande nos Estados Unidos da América (EUA) e no norte europeu, intermediária no leste europeu e na Améri- ca do Sul e baixa na Ásia. Entretanto, a taxa vem aumentando em países com baixa incidência tradicionalmente, como os países asiáticos, particularmente Japão, Cingapura e áreas urbanas da China ${ }^{16}$.

Aproximadamente 180.000 mulheres têm o diagnostico de câncer de mama anualmente nos EUA, sendo responsáveis por aproximadamente $30 \%$ de todos os casos de câncer na mulher ${ }^{16}$ naquele país.

No Brasil, conforme o Instituto Nacional do Câncer ${ }^{17}$, a estimativa para o ano de 2001 é de 4800 casos novos para a região nordeste, 860 destes no estado do Ceará, ocupando, portanto, o segundo lugar, ficando atrás apenas do câncer de pele. A estimativa de óbitos também é elevada, conforme o INCA ${ }^{17}$, e deverá atingir 1190 mulheres na mesma região e 210 no Ceará.

O tamoxifeno é a droga mais comumente prescrita para o tratamento hormonal do câncer de mama. O fármaco é um composto não esteróide que se liga ao receptor estrogênico e atua tanto como agonista quanto como antagonista do estrógeno. Em virtude desta dualidade de ação, agora é referido como modulador seletivo do receptor estrogênico (SERM) ${ }^{16}$.

Vários autores descreveram alterações endometriais em pacientes com câncer de mama tratadas com tamoxifeno ${ }^{15,18-20}$. A propósito, Zarbo et al. ${ }^{18}$, em estudo prospectivo, acompanharam 219 mulheres com câncer de mama tratadas com tamoxifeno, durante seis anos, e descreveram prevalência de $26,9 \%$ de alterações endometriais, representadas principalmente por pólipos e atrofia cística.

No presente trabalho encontrou-se prevalência de $36,7 \%$ de alterações endometriais. Os principais achados foram pólipos em $26,6 \%$ e atrofia cística em 46,6\%. Estes achados estão de acordo com os trabalhos descritos por alguns autores ${ }^{19,20}$. Esta maior prevalência de atrofia cística seria, provavelmente, secundária ao enclausuramento de secreções em tecido glandular endometrial atrófico.

Achados semelhantes foram descritos em coletânea de 55 ensaios clínicos randomizados ${ }^{21}$. A incidência de câncer de endométrio teve incremento de duas vezes com 1 a 2 anos de uso do tamoxifeno e de 4 vezes com 5 anos de uso. Porém, os autores concluem que os beneficios sobrepujam, de forma importante, os riscos da terapia.

Nenhum caso de câncer de endométrio e somente dois casos de hiperplasias simples sem atipias foram encontrados neste estudo. Nestes dois casos a medida da espessura do endométrio foi maior que $10 \mathrm{~mm}$. Embora este estudo envolva somente número limitado de mulheres, reforça a 
necessidade de estudos epidemiológicos que envolvam grande número de pacientes com uso prolongado de tamoxifeno, visto que apenas seis em cada 10.000 mulheres por ano irão desenvolver câncer de endométrio.

Neste sentido foram desenvolvidos vários métodos para investigar a mucosa uterina, entre os quais destacam-se a curetagem uterina, a biópsia endometrial, a ultra-sonografia transvaginal, a histerosonografia e a histeroscopia. Quanto à ultra-sonografia, comprova-se ter importância crescente na propedêutica ginecológica. Constitui método não invasivo, acessivel, inócuo, possibilitando a imediata análise das imagens. O exame transvaginal oferece maior riqueza de detalhes da pelve pelo uso de transdutores de maiores freqüências ( 5 a $7,5 \mathrm{MHz}$ ) e pela proximidade destes às estruturas a serem estudadas. Além disso, a via vaginal elimina a interposição de tecido celular subcutâneo e de alças intestinais. Dos aspectos ultra-sonográficos avaliados, o principal no diagnóstico de anormalidades da mucosa uterina é a espessura do seu eco's .

Há autores que descreveram achado ultrasonográfico de aumento da espessura endometrial em usuárias de tamoxifeno não associado à lesão endometrial, porém relacionado com a atrofia cística $^{23}$. Estudando, por ultra-sonografia, 1010 pacientes na pós-menopausa com câncer de mama em tratamento prolongado com tamoxifeno, Cechini et al. ${ }^{23}$ registraram 351 casos com espessura endometrial maior do que $5 \mathrm{~mm}$, dos quais quatro casos de câncer de endométrio, o que demonstra elevada taxa de falso-positivo do método. Love et al. ${ }^{24}$, utilizando espessura endometrial de $5 \mathrm{~mm}$ como limite para indicação de avaliação histopatológica do endométrio, estudaram 487 pacientes com câncer de mama, das quais 357 haviam sido tratadas com tamoxifeno. Encontraram $41 \%$ de endométrio com aparência ecográfica de anormalidade, dos quais $46 \%$ eram falso-positivos.

Fong et al. ${ }^{25}$, em estudo prospectivo no qual foram incluídas 138 mulheres, empregando ultrasonografia transvaginal comparada com histeroscopia e histopatologia, descrevem, por meio de curva ROC, como ponto de corte ideal para alteração endometrial o valor de $6 \mathrm{~mm}$. Vários autores assinalaram que o aumento da espessura do endométrio à ultra-sonografia transvaginal não necessariamente correspondia à lesão da mucosa uterina, estando a mesma associada a numerosos casos de falso-positivos ${ }^{23,25}$.

Da mesma forma, no presente estudo, utilizando como achado anormal do endométrio espessura maior ou igual a $5 \mathrm{~mm}$, encontrou-se $60 \%$ dos casos como anormais. Mas quando se relacionou o achado ultra-sonográfico com o histopatoló- gico obteve-se taxa de $42,1 \%$ de falso-positivos e razão de probalidade positiva de 2,3 , denotando apenas acurácia moderada, conforme definição de acurácia de Sackett ${ }^{26}$.

Ante ao exposto, provavelmente, numerosas intervenções cirúrgicas desnecessárias foram e serão realizadas, se baseadas apenas no aumento da espessura do endométrio, sem beneficio real para estas enfermas.

A curva ROC mostra a gravidade do contrabalanço entre a sensibilidade e a especificidade e em geral o melhor ponto de corte fica no "ombro da curva" ou próximo dele ${ }^{26}$. No estudo em questão o valor encontrado foi de $8 \mathrm{~mm}$. Utilizando este parâmetro, obtiveram-se sensibilidade de $72,7 \%$, especificidade de $78,9 \%$, valores preditivos positivo de $66,6 \%$, valor preditivo negativo de $83,3 \%$, acurácia de $76,6 \%$, razão de probalidade de 3,4 e taxa de falso-positivo de $21 \%$. Portanto, houve melhora da especificidade, valores preditivo positivo e negativo, diminuindo a taxa de falso-positivos sem comprometer a sensibilidade quando se compara este ponto de corte à medida de $5 \mathrm{~mm}$.

Baseados nestes achados, nos últimos anos, vários estudos ressaltaram o papel da histeroscopia na avaliação da mucosa uterina ${ }^{12,19}$. Pode ser feita ambulatorialmente e sem anestesia. Todavia é exame invasivo, requer técnica especializada e equipamento de alto custo, o que dificulta seu uso para fins de rastreamento populacional.

Gonçalves et al. ${ }^{11}$, em nosso meio, descreveram o seguimento de 46 pacientes, das quais 20 utilizaram tamoxifeno no tratamento do câncer de mama e 26 foram controles. Foram submetidas a histeroscopia antes do início do tamoxifeno e depois de um ano de terapia. Não encontraram diferenças significantes nos grupos de estudo e controle. Os achados histeroscópicos do estudo foram considerados normais em 40,1\% e anormais em $59,9 \%$. Dos casos dentre os achados normais destacou-se a atrofia endometrial. Quando correlacionada com o histopatológico, a histeroscopia demonstrou sensibilidade de $90,9 \%$, e especificidade de $68,4 \%$, com taxa de falso-positivo de $31,5 \%$.

Com estes dados a histeroscopia se apresenta como bom método de rastreamento de alterações endometriais em pacientes com câncer de mama tratadas com tamoxifeno.

Enfim, o tamoxifeno permanece como tratamento endócrino de primeira linha para todos os estágios do câncer de mama e está associado com baixo risco de desenvolvimento de câncer endometrial; o rastreamento de anormalidades endometriais em pacientes menopausadas assintomáticas não apresenta boa relação custobeneficio. 


\section{ABSTRACT}

Purpose: to analyze sensitivity, specificity, positive and negative predictive values and the likelihood ratio of transvaginal ultrasound and hysteroscopy when compared with the histopathologic examination of the endometrium in women with breast cancer who have been treated with tamoxifen.

Methods: transversal study with 30 women in whom transvaginal ultrasound evaluated the echogenic pattern of endometrial echo and its thickness. Hyteroscopy was performed and described as normal (normal or atrophic endometrium) or abnormal (thickening, polyps, leiomyoma, synechia). Material for histopathology was obtained from endometrial biopsy and the findings were considered normal (irregular endometrial maturation and/or atrophy) or abnormal (polyps, simple or complex hyperplasia, leiomyoma or endometrial carcinoma).

Results: the general diagnosis of endometrial modifications was present in $36.6 \%$ of patients. The most frequent results were cystic atrophy (46.6\%) and endometrial polyps (26.6\%). Through the ROC curve the best cutoff of $8 \mathrm{~mm}$ of endometrial thickness measure was determined. This measure showed sensitivity of $72.7 \%$, specificity of $72.9 \%$, positive predictive value of $66.6 \%$, negative predictive value of $83.3 \%$ and likelihood ratio of 3.4. Hysteroscopy showed sensitivity of $90.9 \%$, specificity of $68.4 \%$, positive predictive value of $62.5 \%$, negative prediction value of $92.8 \%$ and likelihood ratio of 2.8 .

Conclusions: the most frequent endometrial modifications were cystic atrophy followed by polyps. Transvaginal ultrasound showed a higher rate of false-positive (42.1\%), when the cutoff for the thickness of the endometrium was 5 $\mathrm{mm}$; however acuracy improved when the measure of $8 \mathrm{~mm}$ was used. The cutoff of $8 \mathrm{~mm}$ was determined through the ROC curve.

KEY WORDS: Tamoxifen. Endometrium. Transvaginal ultrasound. Hysteroscopy. Breast cancer.

\section{Referências}

1. Karck U, Kommoss F. Does tamoxifen change oestrogen and progesterone receptor expression in the endometrium and breast? Eur J Cancer 2000; 36 Suppl 4:S45-6.

2. Elkas J, Armstrong A, Dohl J, Cuttitta F, Martinez A, Gray K. Modulation endometrial steroid receptors and growth regulatory genes by tamoxifen. Obstet Gynecol 2000; 95:697-703.

3. Rodrigues de Lima G, Nazário ACP. Câncer de mama, endométrio e tamoxifeno. Rev Bras Mastol 1996; 6:5-6.
4. Rullo S, Tagliaferri T, Bandiera F, et al. Uterine changes during tamoxifen therapy. Clin Exp Obstet Gynecol 1993; 20:116-9.

5. Gallo MA, Kaufman D. Antagonistic and agonistic effects of tamoxifen: significance in human cancer. Semin Oncol 1997; 24 Suppl 1:S1-71-S1-80.

6. Cole MP, Jones CTA, Todd IDH. A new antioestrogenic agent in late breast cancer: an early clinical appraisal of ICI 46474. Br J Cancer 1971; 25:270-5.

7. Barakat RR. Tamoxifen and endometrial neoplasia. Clin Obstet Gynecol 1996; 39:629-40.

8. Fisher B, Constantino JP, Wickerham DL, et al. Tamoxifen for prevention of breast cancer: report of the National Surgical Adjuvant Breast and Bowel Project P-1 Study. J Natl Cancer Inst 1998; 90:1371-88.

9. Mourits MJ, De Vries EG, Willemse PH, Ten Hoor KA, Hollema H, Van der Zee AG. Tamoxifen treatment and gynecology side effects: a review. Obstet Gynecol 2001; 97:855-66.

10.Nishimura N, Hachisuga T, Saito T, Kawarabayashi T. Subsequent endometrial carcinoma with adjuvant tamoxifen treatment in Japanese breast cancer patients. Int $\mathrm{J}$ Gynecol Cancer 2001; 11:272-6.

11. Gonçalves MA, Gonçalves WJ, Matias MM, Nazario AC, De Lima GR, Baracat EC. Hysteroscopic evaluation of the endometrium of post-menopausal patients with breast cancer before and after tamoxifen use. Int $\mathrm{J}$ Gynaecol Obstet 1999; 66:273-9.

12.Gebauer G, Hafner A, Siebzehnrubl E, Lang N. Role of hysteroscopy in detection and extraction of endometrial polyps: results of a prospective study. Am J Obstet Gynecol 2001; 184:59-63.

13.Fleischer AC, Wheeler JE, Lindsay I, et al. An assessment of the value of the ultrasonographic screening for endometrial disease in postmenopausal women without symptoms. Am J Obstet Gynecol 2001; 184:70-5.

14.Symonds I. Ultrasound, hysteroscopy and endometrial biopsy in the investigation of endometrial cancer. Best Pract Res Clin Obstet Gynaecol 2001; 15:381-91.

15.Gerber B, Krause A, Muller H, et al. Effects of adjuvant tamoxifen on the endometrium in postmenopausal women with breast cancer: a prospective long-term study using transvaginal ultrasound. J Clin Oncol 2000; 18:3464-70.

16.Willet WC, Rockhi B, Hankinson SE, Hunter DJ, Colditz GA. Epidemiology and nongenetic causes of breast cancer. In: Harris JR, et al., editors. Diseases of the Breast. $2^{\text {nd }}$ ed. Philadelphia: Lippincott Williams \& Wilkins; 2000. p.175-220. 
17.Brasil. Ministério da Saúde. Instituto Nacional do Câncer (Inca). Estimativas da incidência e mortalidade por câncer no Brasil. 2001. [acessado 15 out 2001]. Disponivel em <http// www.inca.org.br/epidemiologia/estimativa 2001>.

18.Zarbo G, Caruso G, Zammitti M, Caruso S, Zarbo $R$. The effects of tamoxifen therapy on the endometrium. Eur J Gynaecol Oncol 2000; 21:86-8.

19. Mourits MJ, Van der Zee AG, Willemse PH, Ten Hoor KA, Hollema H, De Vries EG. Discrepancy between ultrasonography and hysteroscopy and histology of endometrium in postmenopausal breast cancer patients using tamoxifen. Gynecol Oncol 1999; 73:21-6.

20.Deligdisch L, Kalir T, Cohen CJ, de Latour M, Le Bouedec G, Penault-Llorca F. Endometrial histopathology in 700 patients treated with tamoxifen for breast cancer. Gynecol Oncol 2000; 78:181-6.

21.Early Breast Cancer Trialists' Collaborative Group. Tamoxifen for early breast cancer: an overview of the randomised trials. Lancet 1998; 351:1451-67.
22.Tesoro MR, Borgida AF, MacLaurin NA, Asuncion CM. Transvaginal endometrial sonography in postmenopausal women taking tamoxifen. Obstet Gynecol 1999; 93:363-6.

23. Cechini S, Ciatto S, Bonardi R, et al. Risk of endometrial cancer in breast cancer patients under long-term adjuvant treatment with tamoxifen. Tumori 1998; 84:21-3.

24.Love CD, Muir BB, Scrimgeour JB, Leonard RC, Dillon P, Dixon JM. Investigation of endometrial abnormalities in asymptomatic women treated with tamoxifen and an evaluation of role of endometrial screening. J Clin Oncol 1999; 17:2050-4.

25.Fong K, Kung R, Lytwyn A, et al. Endometrial evaluation with transvaginal US and hysterosonography in asymptomatic postmenopausal women with breast cancer receiving tamoxifen. Radiology 2001; 220:765-3.

26. Sackett DL. Diagnosis and screening. In: Sackett DL, editor. Evidence-Based Medicine. $2^{\text {nd }} e d$. Edinburgh: Churchill Livingstone; 2000. p.67-93.

\section{COMUNICADO AOS ASSOCIADOS E LEITORES}

Para maiores informações temos à disposição quatro endereços eletrônicos:
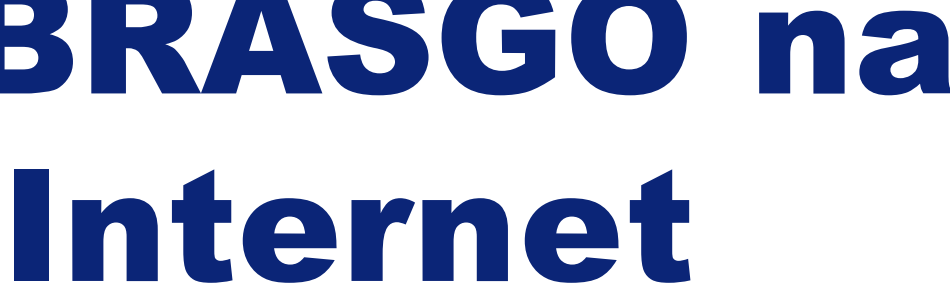

febrasgopresiden@uol.com.br secretaria_executiva@febrasgo.org.br publicacoes@febrasgo.org.br tego_habilitacoes@febrasgo.org.br 